\title{
Editor's Note on the MIC 2013 Special Issue of the Journal of Heuristics (Volume 22, Issue 4, August 2016)
}

\author{
Manuel Laguna ${ }^{1}$
}

Published online: 5 October 2016

(C) Springer Science+Business Media New York 2016

The Journal of Heuristics has implemented a set of policies on heuristic search research. These policies include the Journal's position on Metaphor-based Methodologies. Specifically, the policy states that the Journal of Heuristics avoids the publication of articles that repackage and embed old ideas in methods that are claimed to be based on metaphors of natural or man-made systems and processes. Readers familiar with our policies were probably surprised to see the publication of the article titled "Flying elephants: a general method for solving non-differentiable problems" in the August issue of our journal. This was the result of unfortunate miscommunication with the guest editors of the issue, who somehow were not aware of our policies. For this, I take full responsibility.

Despite the terrible choice for the method's name, in my opinion, the article's content is solid. The method is a generalization and a new interpretation of Hyperbolic Smoothing. The method is shown to perform well in the five problem classes that served as test cases. The authors state that the "name of the methodology, Flying Elephants, is definitely not associated to any analogy with the biology area." This is essentially saying that they have never seen elephants fly, for which I do not feel compelled to require any proof. It is beyond my comprehension why these authors saw the need for this absurd naming of their method. However, this was done and now we have it in the title of one of our articles. Had this article been sent as a normal submission, chances are that it would have been published, but we would have asked the authors to drop any references to flying elephants or any other ridiculous analogy.

I encourage authors that before they submit their work to the Journal of Heuristics they review our policies, which include the journal's position on metaphor-based

$凶$ Manuel Laguna

laguna@colorado.edu

1 Media One Professor of Management Science, Leeds School of Business, University of Colorado at Boulder, Boulder, USA 
methodologies, competitive testing and up-the-wall game, development of customized solutions, and statistically valid experiments and parameter tuning. The policies can be found in the "For Authors and Editors" section of the journal's webpage. 